\title{
Lecturas desobedientes de un sujeto excéntrico: Clara y los pliegues de la subjetividad femenina*
}

*Fecha de recepción: 6 de julio de 2015. Fecha de aceptación: septiembre de 2016

\section{Resumen}

El presente artículo aborda las relecturas y resignificaciones que Luisa Valenzuela realiza sobre ciertos discursos comprendidos como tecnologías sociales, esto es, como agentes configuradores de subjetividad. Partiendo de la lectura atenta de un tango bien famoso, Mano a mano, y espejándolo con algunos de los personajes de Hay que sonreír (1966), se analizarán esas relecturas y resignificaciones que se manifiestan como sabotajes a la letra tanguera, en especial en lo que se refiere a la construcción social de los sujetos femeninos y masculinos.

\footnotetext{
Abstract

This paper deals with the reinterpretations and resignifications that Luisa Valenzuela does on certain discourses that one could understand as social technologies, this is, as agents of subjectivity shaping. Starting with the close reading of the famous tango, "Hand to hand", and comparing with some of the characters of You must smile (1966), we'll analyze these reinterpretations and resignifications that express sabotages of some aspects of the tango, specially those that relate the social constructions of feminine and masculine subjects.
}

\section{Palabras clave:}

sujeto excéntrico discursividad, subjetividad social, sabotaje.

\section{Keywords:}

excentric subject, discourses, social subjectivity, sabotage. 


\section{Lecturas desobedientes de una (posible) realidad}

Rechiflado en mi tristeza, te evoco y veo que has sido en mi pobre vida paria solo una buena mujer. Tu presencia de bacana puso calor en mi nido,

fuiste buena, consecuente, y yo sé que me has querido como no quisiste a nadie, como no podrás querer.

Se dio el juego de remanye cuando vos, pobre percanta, gambeteabas la pobreza en la casa de pensión. Hoy sos toda una bacana, la vida te ríe y canta, los morlacos del otario los jugás a la marchanta como juega el gato maula con el mísero ratón.

Hoy tenés el mate lleno de infelices ilusiones, te engrupieron los otarios, las amigas y el gavión; la milonga, entre magnates, con sus locas tentaciones, donde triunfan y claudican milongueras pretensiones, se te ha entrado muy adentro en tu pobre corazón.

Nada debo agradecerte, mano a mano hemos quedado; no me importa lo que has hecho, lo que hacés ni lo que harás... Los favores recibidos creo habértelos pagado $y$, si alguna deuda chica sin querer se me ha olvidado, en la cuenta del otario que tenés se la cargás.

1 Se trata del famoso Mano a mano, escrito por Celedonio Flores y musicalizado por Carlos Gardel y José Razzano.
La letra de este tango ${ }^{1}$ reconstruye, desde la mirada despectiva de la voz masculina, los espacios en los que se desarrolla el decadente presente de una novia de la juventud. Espacio urbano, el de una Buenos Aires milonguera que a principios de la década de 1920 se ha transformado ya en la metrópolis espejo de las grandes ciudades modernizadas de América Latina. Pero también espacio íntimo, el de una pareja con un pasado de pobreza, prostitución y abnegación femenina, que en el presente se ve plasmado en el despecho de esta voz masculina con un discurso contradictorio y lleno de "entre líneas": nos dice que ha sido y es una buena mujer, y a pesar de ello nos dibuja una imagen distorsionada de ella, en la que sus ilusiones han terminado en un fracaso y en la que su presente sigue estando marcado por el juego y la prostitución. Tal y como supo ver en su momento Sonia Mattalía (2003), ello es consecuencia de un cambio de paradigma en la configuración social y urbana de nuevos sujetos femeninos: "Entrar en el juego de miradas deseantes de la urbe moderna trae consigo un cambio del lugar de los cuerpos femeninos que, como las ropas exhibidas en los escaparates de las grandes tiendas, se miden y estiman por su reificación mercantil" (Mattalía, 2003: 142). 
El valor que este tango otorga al cuerpo evidencia una concentración de miradas sobre el mismo ya que del declive físico de este amante despechado no se habla en ningún momento. Es más: la imagen que de él se representa es la de un ser dolorido pero conmovido por el ocaso de su ex amante, a la que le desea todo lo mejor y a la que incluso le ofrece un último apoyo, en un gesto no exento de cierto heroísmo.

Apenas unos cuarenta años más tarde, este tango aparece citado en Hay que sonreír (1966), ${ }^{2}$ la primera novela de Luisa Valenzuela, junto con otros dos: El Choclo, de alabanza al tango, y Che, Amarroto, de burla al hombre sometido a la voluntad de la mujer. Lo curioso es que Mano a mano es el único en el que Clara, la protagonista, no logra escuchar en boca de su amante, distraída como está en sus propios pensamientos: Cuando yo era chica portarse bien quería decir ayudarla a mamá a baldear el piso, lavar los platos, y también ir todos los domingos a misa. Después portarse bien era no dejar que los muchachos me besaran en las calles oscuras y retarlos cuando me manoseaban. ¿Qué demonios querrá decir portarse bien ahora? (Valenzuela, 2007: 89). Teniendo en cuenta la historia personal de Clara, prostituida casi sin querer por las circunstancias y por su pasividad, y teniendo en cuenta también que el origen de dichas reflexiones se encuentra en un comentario de Monona, la otra prostituta de la historia ("Ahora me prometió [el Cacho, su proxeneta] que si me porto bien me lleva este fin de semana a Mar del Plata") (p. 89), es fácil entrever el componente irónico de estos pensamientos en la chica, así como el carácter casi crítico de la pregunta final. ¿Qué es portarse bien para una chica normal en la Argentina en la década de 1950? Hacer las tareas del hogar, ir a la iglesia y no dejarse manosear por los muchachos, tres instancias que ella no logra cumplir ni siquiera cuando tiene la oportunidad. De hecho, mientras dura su relación con Víctor, el hombre que le ofrece una vida como ángel del hogar, sigue ejerciendo su oficio sin dudarlo, aunque "... después su vocación de ama de casa [gane] la partida y en lugar de comprarse la combinación de puntillas que tenía entre ceja y ceja [vuelva] a la casa con un pollo y una buena botella de vino" (p. 29).

Clara es, a todas luces, como la ex amante de Mano a mano y, sin embargo, el hecho de que no llegue a escuchar la letra de la canción de boca de su nuevo amante le permite desviarse un poco del encorsetado modelo que esta convoca. Y digo un poco porque, al igual que la mujer del tango, ella sigue prostituyéndose de forma más o menos velada y tiene también el mate lleno de ilusiones truncadas. Lo que la distingue de ella es que en el gesto final del abandono de Carlos, de manera bien sutil Clara le devuelve al hombre un reflejo distorsionado de su construcción social. Tiene razón Linda Craig cuando afirma que "[e]l poco tiempo que pasa junto a Carlos es interesante en parte porque su rechazo por no ser lo suficientemente dominante y por eso mismo no aprovecharse del estereotipo del macho de la cultura popular, hace que decida dejarlo finalmente" (Craig, 2005: 117-118), ${ }^{3}$ pero me parece importante recalcar que en dicha actitud Clara está poniendo de manifiesto los pliegues y dobleces por los que las representaciones genéricas se desbordan y acaban por anular su significación social y discursiva.

La mención que Clara hace del discurso materno no me parece, en este sentido, gratuita, sobre todo si tenemos en cuenta que la misma división a la que alude el tango aquí citado entre el espacio externo -una Buenos Aires milonguera y cosmopolita-y el espacio interno -dos amantes con distinto recorrido vital pero con similar recorrido urbano- tiene su reflejo en la división maniquea de dos tipos de mujer: por un lado, la milonguita, una chica de barrio -o de pueblo, como es Clara- que abandona su hogar enceguecida por las luces del centro/ciudad y que, haciéndose pasar por quien no es o dejándose llevar por el rápido movimiento de la metrópoli, comienza un vertiginoso camino de ascensos y descensos espirituales y físicos. Y, por el otro, la madre, figura que condensará el polo opuesto a esta mujer mundana, y que se revestirá de un aire
2 En el cuerpo del texto, me voy a limitar a citar solo las páginas correspondientes a la edición de Valenzuela, 2007.

3 De mi traducción. Así en el original: "The short time that she spends with Carlos is interesting partly because of her rejection of him for not being of her rejection of him for not being
sufficiently dominating and therefore not living up to the macho masculine stereotype of popular culture; indeed she decides to leave him ultimately". 
4 A pesar de ello, y como bien señala Karina Felitti, "la maternidad y la vida hogareña continuaban ocupando un lugar privilegiado" (Gil Lozano, et al. (2000, 18) todavía en la época en te transcurre la novela (finales de la década de 1950, principios de la década de 1960, si tenemos en cuenta que este es e momento de escritura de la misma). de misticismo y abnegación. Y no creo que sea en vano su recuerdo porque si en algo se caracteriza la madre de Clara es, precisamente, en no ser imagen de nada o, mejor, en ser la imagen de una mujer ausente de su hogar, a pesar de que en el recuerdo de la chica conserva un lugar de privilegio:

Mi madre, que sabía lo que eran buenos modales porque iba a lavar y planchar a lo de Brunetti, gente de lo mejor, nunca colgó esas tiras inmundas. Pero cuando se fue con los dos menores a pasar esas vacaciones a Quemú-Quemú en casa de tía, esas vacaciones que no terminaron más, entonces padre se dejó convencer por las vecinas - por la mujer del carnicero, ahora sí que lo sé-y colgó unas tiras larguísimas de una pared a la otra de la cocina. (Valenzuela, 2007: 57; la cursiva es mía)

Reproduzco casi por entero el discurso de la chica porque me parece bien sintomático de lo que nos encontramos a lo largo de la novela: un texto tético-el discurso de Clara- que, al generar una antítesis conceptual, crea un modelo de mundo que en vez de incitar al diagnóstico positivo de la madre -propósito de la protagonista-, provoca en nosotros el efecto contrario. El juego antitético entre los buenos modales de una mujer que se gana la vida como empleada doméstica y su ausencia del hogar no solo producen un rechazo a la carga perceptivo-ideológica de las palabras de Clara sino que, por un efecto retroactivo, ameritan la resignificación especular de los conceptos que ella misma ha dado en emplear. Y es que, según lo que podemos inferir de sus palabras, una buena mujer ya no es la que se queda en el hogar y explota su devenir sujeto maternal sino más bien la otra, esa que sale a conquistar el mundo y que va ganando terreno como sujeto social.

Afirma Sonia Mattalía que los rápidos procesos de urbanización y democratización que afectaron sobre todo a las principales ciudades de América Latina desde finales del siglo XIX conllevaron el avance de la mujer hacia espacios de carácter público como el trabajo y la educación:

Sobre el cambio del viejo perfil de las ciudades coloniales, abiertas ahora por las grandes avenidas según el modelo de Haussmans, con sus agitados centros comerciales, se recortan las imágenes de una nueva mujer: empleadas de servicio, amas de casa, maestras, oficinistas, dependientas, obreras y algunas profesionales, las mujeres ganan la calle. (Mattalía, 2003: 143; el último subrayado es mío) ${ }^{4}$

Ciudad y mujer conforman así un binomio interesante en el que se mezclan particularidades de carácter público y privado, pero también, y muy especialmente, cuestiones de orden político e ideológico: "la mujer se transforma en un sujeto social que empieza, a la par, a integrarse en el mundo laboral y a estructurar sus propias plataformas reivindicativas" (Mattalía, 2003: 143).

Para concluir este primer apartado, podemos decir que Luisa Valenzuela desvirtúa el discurso tanguero y, además, se atreve a dibujar personajes que se desvían de su norma. Seguramente porque, como ella misma ha señalado en otro lugar, “... me interesa deconstruir el discurso del poder, ver a través de él, develar qué nos está diciendo en realidad mientras expresa aparentemente todo lo contrario." (Valenzuela, 2001: 90). Y a nadie se le escapa el poder discursivo del tango en una sociedad que avanza en sus modificaciones urbanas y en sus transformaciones sociales, pero que necesita seguir delimitando ciertas fronteras, al menos entre los individuos que la habitan. Como discurso difusor del modelo de mundo del pensamiento hegemónico -y léase, aquí, patriarcal-, el tango aspira a perpetuar viejas estructuras dentro de la modernización, y ello afecta en especial al modelaje de los sujetos femeninos y masculinos. 
Su papel, en este sentido, es casi tan importante como el que los libros jugaron apenas un siglo antes: abrir las posibilidades -o imposibilidades- ontológicas del devenir sujeto social y develar las falacias de las mismas. No en vano, el encuentro del mundo de Clara con el mundo de los tangos en su visita nocturna a Carlos se extiende fuera de sí mismo a través de las representaciones de los tres tangos citados. Y es que, tal y como Nora Catelli señalara en su momento a propósito de las figuras de la lectura, “... son pruebas, 'testimonios tangibles' de la lectura (hubiese dicho Lucien Fevbre), pero, al mismo tiempo, 'testimonios tangibles' de su precariedad, de su fragilidad: muestran los proyectos, promesas, sueños o temores que ella suscita" (Catelli, 2001: 196). Si ahí donde la argentina habla de figuras de la lectura ponemos figuras de la escucha, podemos aventurar que en Hay que sonreír la representación de una escena de escucha -Clara queriendo y no pudiendo escuchar Mano a mano de boca de su amante- es también el testimonio más tangible -por más claro y evidente- de que lo representado, el tango en la novela y lo que este intenta transmitir fuera ya de los límites de ella, no son más que el producto de una modelización previa.

Con ello quiero decir que la imagen de un hombre joven cantando un tango cuya letra denota ciertas representaciones socioculturales con el único fin de perpetuarlas y naturalizarlas en quienes quieran escucharlas (por ejemplo, la misma Clara), nos enfrenta al hecho insoslayable de que todo discurso compromete una acción modelizadora, esto es, una acción por medio de la cual los sujetos que perciben, conciben y se representan el mundo y a sí mismos de una manera codificada de antemano son inter(a)pelados, incitados e, incluso, performados en su devenir sujetos sociales (Asensi Pérez, 2011). Cuando, a propósito de estas figuras femeninas que el tango en general dibuja, Raúl Horacio Campodónico y Fernanda Gil Lozano afirman: "Madre e hija son acciones: lavar, sufrir y esperar para la primera; transitar la noche y el cabaret, beber champán, lucir joyas y pieles para la segunda. El discurso de ellas es meramente gestual, corporal" (Gil Lozano et al., 2000, 2: 144), están avanzando en esta misma dirección, si bien en el caso de Clara el discurso meramente corporal tendrá su contraparte en un lenguaje interior que muchas veces funcionará como una especie de salvavidas emocional.

Pensemos, si no, en la división meta-textual de la novela en tres apartados: Cuerpo, Transición y Cabeza. A ello, añadámosle las palabras de Luisa Valenzuela, para quien escribir con el cuerpo supone "... un trabajo conjunto de la cabeza y lo otro, aquello que nos impulsa a movernos, a bailar y respirar y exhalar, es decir por lo tanto acarrea a la cabeza y con ella al raciocinio a explorar caminos impensados que suelen asustarnos" (Díaz; en Díaz y Lagos, 1996: 29). Y ahora cerremos con estas consideraciones de Alejandro, el último de los hombres con los que se relaciona Clara en su largo periplo de descenso a los infiernos de la subjetividad: "Para dominarla, para absorberla por completo no bastaba con dejarla sola en una pieza de hotel, abandonada. Clara encontraria siempre una escapatoria para pensar en otra cosa, para no dejarse aplastar por él." (Valenzuela, 2007: 144; la cursiva es mía).

Esta capacidad de Clara de "pensar en otra cosa" es lo que, por un lado, la salva de languidecer al lado de los hombres que elige para seguir camino y, por el otro, la aleja definitivamente del modelo tanguero, en tanto que ella sí tiene una voz. Quizá esta no sea explícita, quizá las más de las veces aparezca ninguneada o burlada por aquellos que la rodean, como cuando Víctor le espeta: “'Acaso te obligan a decir idioteces cada vez que abrís la boca?" (p. 54), pero lo cierto es que Clara habla, y mucho, sobre ella, sus deseos y sus expectativas:

Clara fregaba mucho sin decir una palabra porque él no admitía réplicas y ella ya había perdido el uso de su voz aunque manejaba con destreza su imaginación. Su diálogo mental favorito era el del matrimonio, lo había perfeccionado y no sólo estaba segura 
de su indiscutible realismo, sino que al final no había vencedores ni vencidos, ya que ella tampoco tenía muchas ganas de casarse con Víctor. Los días que no tenía nada que hacer lo estiraba hasta el infinito, en cambio cuando estaba cansada lo resumía en pocas palabras hasta llegar a la conclusión de que no valía la pena casarse ni tener hijos porque, como decía Víctor, eso es Ilenarse de complicaciones. (Valenzuela, 2007: 29; la cursiva es mía)

La cita es bastante representativa del modo de pensar-actuar de Clara a lo largo de la novela. En una separación que poco a poco va a ir sobreponiéndose, Clara abandona el cuerpo al exterior y lo ofrenda a quien quiera adueñarse de él ("Iría lúcida al sacrificio -se nos explica apenas unas páginas antes-, como esas vacas que había visto llevar al matadero y que sin lugar a dudas sabían que iban a morir") (p. 18), pero conserva en un lugar de intimidad a la cabeza y a lo que esta pueda aportarle. Lo interesante, sin embargo, es que más que raciocinio, esta la enfrenta constantemente con lo condicional, de manera que su historia no es más que un ir y venir entre ilusiones y frustraciones. Por otro lado, nótese al final de la extensa cita cómo la referencia al pensamiento de Víctor le otorga cierta legitimidad a su discurso y se la quita, en cambio, al suyo, en un ejemplo que se repite a lo largo de la novela y que nos invita a plantearnos el lugar que ocupa ella como sujeto.

\section{Primer pliegue: la configuración resistente de un cuerpo}

En este marco de desobediencias, pues, la figura de la muchacha se recorta sobre una Buenos Aires sucia, ruidosa y superpoblada, para insertarse en la vorágine de cambios y reacomodamientos. Como estas mujeres a las que alude Sonia Mattalía, Clara llega para ganarse la calle, y lo hace en un sentido metafórico y en uno literal: nada más bajarse del tren lo primero que busca es el bosque de Palermo, ya que le han dicho que es lo más lindo de la capital “... con los lagos, los cisnes y un rosedal tan bien cuidado" (Valenzuela, 2007: 14), y a pesar de ello frente a lo que se queda fascinada es ante "... una vidriera con blusas de seda y puntilla y polleras acampanadas" (p. 14). Asimismo, y por una suerte de ironía del destino, Clara se convierte en prostituta, en comerciante de su propio cuerpo.

No es casual, en este sentido, que la primera de las tres partes en las que se divide la novela se titule "El cuerpo", ni que desde sus primeras líneas este sea el protagonista indiscutible de la misma. Como ya intuyó en su momento Sharon Magnarelli, en este comienzo: "Qué opio esperar. Con el pie izquierdo se rascó la pierna derecha en un gesto que quería decir resignación" (Valenzuela, 2007: 11), se esboza una semiótica del cuerpo en la que algunos gestos comunican determinados significados, aun cuando estos puedan variar según el contexto en el que se manifiesten (Magnarelli; en Díaz y Lagos, 1996: 54).

Lo que no vio la crítica norteamericana en ese momento es que la relación significantesignificado no es solo unidireccional y contextual, sino que la mayoría de las veces se manifiesta multidireccional y paradójica. Quizá porque, como ella misma ha apuntado en más de una ocasión "[s]omos manipulados por el lenguaje pero no tenemos otra forma de acceso. Así que hay que jugar con el gran manipulador y develar la multiplicidad de códigos" (Díaz; en Díaz y Lagos, 1996: 46). Esto, en el universo de Luisa Valenzuela, implica aceptar que el lenguaje siempre dice lo que dice y algo más. Y en el hueco que abre este "algo más" es donde se coloca un cuerpo que no solo es semiosis ilimitada de huellas que se mueven continuamente y se resignifican sin descanso, sino también escritura microscópica que informa y configura activamente 
subjetividades. Aunque desde un punto de vista lógico y normativo su significación sea incomprensible y abyecta (Asensi Pérez, 2008).

Si como comenta Luisa Valenzuela: "Allí donde el cuerpo está escribiendo en libertad escribe la metáfora. O, para decirlo de otra forma, se accede al orden de lo simbólico y esa es la búsqueda y esa es la lucha" (Valenzuela, 2001: 133), habrá que tener en cuenta entonces el vínculo problemático entre el cuerpo y el lenguaje o, mejor aun, entre el cuerpo y las palabras, esas partículas ínfimas que, cual cuarkes, están hechas de materia " $\tan$ pero tan pequeñas que carecen de tamaño, pero tienen -por imprescindible convenci- sabor y color, y se presentan intercambiablemente como partícula o como onda, según el ojo del experimentador. O de la experimentadora" (Valenzuela, 2001: 28). En este sentido, el gesto con el que se da comienzo a la novela no puede ser más significativo: Clara se rasca la pierna derecha con el pie izquierdo, en una actitud que, tal y como se nos dice en el relato, denota resignación. Sin embargo, unas páginas más adelante, cuando ya se nos ha informado un poco sobre el tipo de vida que ha llevado la muchacha hasta llegar al punto donde la encontramos, otro es el mensaje que se decodifica. Y es que el cuerpo no es solo el gesto que lo escribe y lo describe, sino una escritura que comunica e impone sus consignas. Aun cuando estas puedan ser (re)interpretadas y (re)significadas desde múltiples y variados ángulos. ¿Cómo entender, si no, que la misma escena-Clara esperando sola en una esquina incómoda de una incómoda y ruidosa Buenos Aires- sea releída desde otro lugar por aquellos transeúntes que la cruzan? La voz narradora es explícita: “... cuando una mujer espera sola en una esquina en lugar de compadecerla los muchachones le gritan: // -iVamos morocha, no ves que el fulano te dejó plantada! Te colgó la galleta, que le dicen..." (Valenzuela, 2007: 47). Y lo que los muchachones gritan, la gente calla con la palabra pero dice con la mirada de reojo: que una mujer espere sola a alguien en una esquina perdida de la ciudad solo puede decir que es una prostituta, es decir, un sujeto abyecto y reprobable.

Comenta Julia Kristeva que "[1]o abyecto es perverso ya que no abandona ni asume una interdicción, una regla o una ley, sino que la desvía, la descamina, la corrompe" (Kristeva, 2004: 25). En el caso de Clara esto es evidente si tenemos en cuenta una vez más el mandato que pesa sobre la mujer en la Argentina a finales de la década de 1950: ser perfecta ama de casa, devota y recatada. Ahora bien, no menos cierto es que esta escena alargada en el tempo narrativo recrea una corrupción de la corrupción primera, que no es otra que la de un lenguaje “... camaleón que cambia de color según las personas que [lo] usan" (47-48), de manera que lo que se representa no es solo una abyecta -por incomprensible e indescifrable- corrupción de la normativa que exige de la mujer dicha actitud, sino también, y muy especialmente, la corrupción corporal de una corrupción lingüística asumida y naturalizada por todos y todas. La conclusión parece fácil de intuir: en el fondo de lo corrupto lo que late es la ley, el logos, de manera que ponerla en entredicho es posible cuando el cuerpo de Clara se escapa de su control y queda a la deriva, sin un sujeto dialéctico de mirada o, mejor, con un sujeto vacío que, en su mirar, descubre una visión velada cuya verdad es velar la nada, la falta fundante de todo ser.

\section{Segundo pliegue: la desobediencia como excentricidad}

Y como vacío es el ser, múltiples son las posibilidades de configuración del mismo. Por eso es que en este apartado propongo una lectura contaminante del sujeto Clara a partir de la lectura de la noción de sujeto excéntrico propuesta por Teresa de Lauretis (2000). 
En primer lugar, porque a todas luces Clara se des-identifica de ese modelo femenino que el tango reivindica y que, como modelo que es, se manifiesta arquetípico y vaciado de cualquier contenido.

En segundo lugar, y en estrecha relación con esto último, porque su des-identificación no implica una identificación con el modelo opuesto, esa masculinidad también puesta en entredicho a lo largo de la novela por las constantes alusiones a su vacuidad: "Clara se alzó de hombros. Estaba asombrada de ver hasta qué punto le resultaban indiferentes los arranques de furia de Víctor" (Valenzuela, 2007: 65) , "Gritaba tanto y resonaba tan bien que Clara empezó a sospechar que estaba hueco por dentro, como un bombo" (p. 45), se nos dice a propósito de Toño; o por las menciones a cierta violencia gratuita e inexplicable, como la que constantemente demuestra Alejandro con Clara, solo porque "[t]enía la filosofía de golpear primero" (p. 110), o como la que antes que él ha manejado Toño Cruz con la chica solo porque piensa que "... lo que había que hacer con las mujeres era tratarlas mal" (p. 45); e incluso por las múltiples referencias a la hipocresía de su moralidad, congelada en una serie de acciones que podemos considerar casi de ritual.

Este último aspecto queda bien representado en el dibujo casi ridículo de un Carlos dominado por el deseo de posesión física de Clara, a pesar de que él, como cantor de tangos que es, se quiere dueño de ciertas legitimaciones emotivas y moralizantes: le prohíbe a Clara relacionarse con "... esa bruja de Monona" y con el Cacho, su proxeneta, pero no duda en empujarla a la casa de citas y, casi sin mediar palabra, desvestirla desesperadamente para hacerle el amor a las apuradas (p. 94). Junto a él, Víctor se perfila también como el vocero de cierta moralidad acartonada y vacía de contenido al sentirse acosado "... por una multitud de prostitutas que trataban de arrancarle la billetera" (p. 56) y al pretender que Clara se comporte como "... una verdadera ama de casa" (p. 28), sumisa, callada y dispuesta a recibir malos tratos cuando las cosas no anden bien: "cuando todo marchaba sobre rieles la llamaba Clarita y la llevaba a pasear. En cambio, cuando surgía alguna dificultad, decía que la culpa la tenía ella por no saber manejar la economía doméstica y la llamaba mi esposa" (p. 29).

En tercer lugar, porque la subjetividad de Clara va construyéndose de una manera bien contradictoria, múltiple y heterogénea. Contradictoria porque, como ya vimos apenas unas líneas más atrás, muchas veces asume el discurso del hombre que tiene al lado en lo que parecería ser un acatamiento total a ciertos discursos, en especial aquellos que la relegan a mero cuerpo mercantilizado. No obstante, su actitud corporalmente pasiva pero mentalmente activa, así como el hecho de que se le dé un espacio bastante amplio a lo largo de la narración para que dé rienda suelta a sus ilusiones, la llevan a negar ese mismo discurso anteriormente aceptado e, incluso, a combatirlo. Por eso, por ejemplo, acaba casándose con Alejandro, el único hombre que se lo pide, porque como ella misma se ve obligada a reconocer, “... siempre había soñado con casarse" (p.132); y por eso también sufre una suerte de extrañamiento cuando lo hace: "Ahora en cambio ajena a todos, la esposa del mago, una cualquiera. Casi una extranjera, mal vista por los de adentro de la kermés y por los que nada tenían que ver con ella" (p. 140).

Estas mismas contradicciones son lo que acompaña también una construcción subjetiva múltiple del personaje, quien, a pesar de todo, se posiciona y es posicionado en un lugar propio e infranqueable. Que este sea el de la imaginación -la loca de la casatiene que ver con la necesidad de explorar los caminos significantes de la nada, es decir, tiene que ver con una voluntad explícita de sostener lo que no existe por medio de un cuerpo, sí, pero también, y muy especialmente, de las palabras. A ello se refiere Luisa Valenzuela en cierta ocasión, cuando comenta: 
No se puede separar el logos del cuerpo [...]. Y ahí es donde perdés el poder, no te importa, porque ahí la palabra no va a ser para dominar al otro tanto como para decir tu esencia, expresar tu deseo. Y ese decir tu deseo es muy difícil porque, como sabés, el deseo no quiere ser dicho, entonces va a luchar y esa es una manera de dominar al otro, que el otro diga su deseo. (Díaz; en Díaz y Lagos, 1996: 32)

Teniendo esto bien presente, creo que es más fácil comprender el último de los vínculos generados por la chica con Alejandro, así como la simbiosis absoluta que se establece entre ambos: "Desde el otro cuerpo le llegaba una intensa corriente que la sacudía, que no le daba paz, que le quitaba todo el bien que podía venir desde afuera porque lograba que adentro de ella las aguas estuvieran revueltas" (Valenzuela, 2007: 159). Si recordamos, Alejandro arde en deseos de dominar a Clara por completo y, para ello, sabe que no solo debe conquistar el terreno corporal, sino sobre todo el mental, pues es ahí donde siente que ella lo domina, al empujarlo a enfrentar sus propias contradicciones, sus verdades más profundas y sus más oscuros secretos: "Esa cabeza confiada apoyada sobre su hombro lo poonía nervioso. Ella era buena; él la obligaba a llevar una vida de perros y en lugar de disculparse la agobiaba con reproches. Qué injusto, qué injusto, pero no podía evitarlo. Otros debían pagar lo que él hubiera podido ser y no era." (Valenzuela, 2007: 159).

\section{Tercer pliegue: la (im)posibilidad de una subjetividad completa}

"Allí donde el cuerpo está escribiendo en libertad escribe la metáfora" (Valenzuela, 2001: 133), nos dice Luisa Valenzuela, y es en este sentido, en el metafórico, como considero que hay que entender tanto la narración completa de la historia de Clara -sometida corporalmente a los deseos masculinos, pero manteniendo cierta libertad gracias al uso sin cesuras de su cabeza-, como la última de las tres partes del relato.

La historia de la flor azteca pone de manifiesto la sutil maniobra orquestada por Alejandro para dominarla, desgajando el cuerpo de la cabeza, obligándola a ella, que hasta ese momento había mantenido cierto equilibrio entre ambos, a sentir las consecuencias de la fisura: "Ahora el bestia ese quería que todos la vieran como era de verdad, con un cuerpo miserable que ya no quería mostrar todo encorvado dentro de un cajón, con las piernas separadas por un ángulo de espejos..." (169). Asimismo, evidencia los claroscuros de un develamiento cuyo fin no parece ser otro que el de mostrarnos “... los bajos fondos propios del ser humano, del alma” (Valenzuela en su entrevista con Díaz; en Díaz y Lagos, 1996: 46).

En una de las "Ventanas" que pueblan su libro de ensayos Peligrosas palabras-reflexiones de una escritora-, precisamente la que viene a cerrar un texto titulado "Escribir con el cuerpo", Valenzuela se pregunta: “¿El cuerpo como máscara de la mente, o viceversa?" (Valenzuela, 2001: 138). A lo que no duda en responder: "Más bien el cuerpo como máscara del alma" (Valenzuela, 2001: 139). Pues bien, mucho de máscara tiene el cuerpo representado en esta novela, por eso mismo, cuando se lo quitan, lo único que le queda a Clara es realizar(se) en una simbiosis absoluta con Alejandro, ese ser que la maltrata y la humilla porque sí, por la pura gratuidad del hecho, aunque en alguna que otra ocasión de la novela se nos dé a entender que su actitud es fruto de una terrible frustración como sujeto social ("La casa de sus padres en Belgrano, el premio al mejor proyecto para un barrio funcional, aquella Inés que había roto su noviazgo cuando lo echaron de la facultad, el día que se fue de su casa para buscar un nuevo camino, todo estaba demasiado lejos", 2001, 113). Es entonces, cuando se queda sola con su cabeza sin un cuerpo que mostrar y ofrecer, sin un cuerpo que la enmascare ante la sociedad y la signifique reescribiéndola cuantas veces le haga falta, 
que Clara se desarma y se pierde en el discurso de Alejandro, en su odio sostenido a lo largo del tiempo y de las palabras: "Lo había odiado muchas veces, sí, pero nunca como esta. Era algo más grande que ella, que la sobrepasaba. Era tan inmenso su odio que tenía miedo que hiciera estallar los espejos; por eso se contenía, tratando de calmarse" (Valenzuela, 2007, 170; la cursiva es mía).

El mismo odio que Alejandro pone en práctica, asumido genuinamente por ella, sin diferencias, con la misma intensidad aunque sin el mismo dominio pues ya no se trata de asumir el discurso del otro, como ha venido haciendo a lo largo de su vida, sino de algo mucho más profundo y, por lo mismo, más enajenante, más perturbador, en definitiva, más fragmentador: Es mi destino, después de todo [se confiesa Clara en la última página]. No vale la pena escapar, ya, ni gritar ni defenderme. Voy a ser la cabeza sin cuerpo, sin trucos ni espejos. Y mi cabeza va a estar sobre una mesa de verdad bajo la que va a pasar Alejandro con el cuerpo negro y peludo de Asmodeo. (Valenzuela, 2007: 176; la cursiva es mía).

Una cabeza sin cuerpo y un cuerpo sin cabeza para quien ha ido escribiendo con el cuerpo aun sin saberlo, con el compromiso de no huir de aquellos impulsos que debilitan al raciocinio sino más bien todo lo contrario, aceptándolos e integrándolos en la lenta y a veces penosa configuración de su subjetividad. Tiene razón Sharon Magnarelli cuando sostiene que "para Valenzuela, escribir con el cuerpo es escribir de un modo comprometido, activo y participativo, es escribir lo que uno ha experimentado, aun cuando se reconozcan las limitaciones de las interacciones del cuerpo con el mundo exterior" (Díaz y Lagos, 1996: 69). Pero no es menos cierto que el compromiso a veces puede transcurrir por otros derroteros. Clara lo deja bien claro cuando, a las miles de contradicciones y multiplicidades de su ser sujeto, les añade la particularidad de lo heterogéneo, ya que su posicionamiento la lleva a experimentar en sus carnes la travesía de distintas prácticas y discursos que la determinan y la per-forman. 


\section{Q Bibliografía}

"Asensi Pérez, Manuel (2008). "El poder del cuerpo o el sabotaje de lo construido", en Encarna(c)ciones. Teoría(s) de los cuerpos, Barcelona, UOC.

"Asensi Pérez, Manuel (2011). Crítica y sabotaje, Barcelona, Anthropos.

"Catelli, Nora (2001). Testimonios tangibles. Pasión y extinción de la lectura en la narrativa moderna. Barcelona, Anagrama.

"Craig, Linda y Onetti, Juan Carlos (2005). Manuel Puig and Luisa Valenzuela: Marginality and gender, Woodbridge, Tamesis.

"De Lauretis, Teresa (200o), Diferencias. Etapas de un camino a través del feminismo, Madrid, Horas y horas.

"Díaz, Gwendolyn, “Entrevista con Luisa Valenzuela. EmoryUniversity, Atlanta, 1994", en Díaz, Gwendolyn y Lagos, María Inés (editoras), La palabra en vivo: la narrativa de Luisa Valenzuela, Santiago de Chile, Editorial Cuarto Propio, págs. 27-52, 1996.

» Felitti, Karina (2000). El placer de elegir. Anticoncepción y liberación sexual en la década del sesenta", en Historia de las mujeres en la Argentina, tomo II: Siglo $X X$, Buenos Aires, Taurus.

" Gil Lozano, Fernanda y Campodónico, Raúl Horacio (2000). “Milonguitas encintas. La mujer, el tango y el cine", en Historia de las mujeres en la Argentina, tomo II: Siglo XX, Buenos Aires, Taurus.

» Kristeva, Julia (2004). Poderes de la perversión. Ensayo sobre Louis-Ferdinand Céline. Trads. Nicolás Rosa y Viviana Ackerman. México, Siglo XXI.

»Magnarelli, Sharon (1996). "Luisa Valenzuela: Cuerpos que escriben (metonímicamente hablando) y la metáfora peligrosa", en La palabra en vivo: la narrativa de Luisa Valenzuela, Santiago de Chile, Editorial Cuarto Propio.

"Mattalía, Sonia (2003). Máscaras suele vestir. Pasión y revuelta: escrituras de mujeres en América Latina, Madrid / Frankfurt, Editorial Iberoamericana / Vervuert.

»Valenzuela, Luisa (2001). Peligrosas palabras -reflexiones de una escritora-, Buenos Aires, Temas.

»---------- ([1966]2007). Hay que sonreír, Buenos Aires, Fondo de Cultura Económica. 
